\title{
Hydrogenation Facilitates Proton Transfer Through Two-Dimensional Honeycomb Crystals
}

\author{
Yexin Feng, ${ }^{1, *}$ Ji Chen, ${ }^{2, *}$ Wei Fang, ${ }^{3}$ En-Ge Wang, ${ }^{4}$ Angelos Michaelides,,${ }^{2, \dagger}$ and Xin-Zheng Li ${ }^{4}$, \\ ${ }^{1}$ School of Physics and Electronics, Hunan University, Changsha 410082, P. R. China \\ ${ }^{2}$ Thomas Young Centre, London Centre for Nanotechnology, and Department of Physics and Astronomy, \\ University College London, London WC1E 6BT, United Kingdom \\ ${ }^{3}$ Thomas Young Centre, London Centre for Nanotechnology, and Department of Chemistry, \\ University College London, London WC1E 6BT, United Kingdom \\ ${ }^{4}$ School of Physics, ICQM, and Collaborative Innovation Center of Quantum Matter, \\ Peking University, Beijing 100871, P. R. China
}

(Dated: September 29, 2018)

\begin{abstract}
Recent experiments have triggered a debate about the ability of protons to transfer through individual layers of graphene and hexagonal boron nitride (h-BN). However, calculations have shown that the barriers to proton penetration can, at more than $3 \mathrm{eV}$, be excessively high. Here, on the basis of first principles calculations, we show that the barrier for proton penetration is significantly reduced, to less than $1 \mathrm{eV}$, upon hydrogenation even in the absence of pinholes in the lattice. Analysis reveals that the barrier is reduced because hydrogenation destabilises the initial state (a deep-lying chemisorption state) and expands the honeycomb lattice through which the protons penetrate. This study offers a rationalization of the fast proton transfer observed in experiments, and highlights the ability of proton transport through single-layer materials in hydrogen rich solutions.

PACS numbers: $68.43 . \mathrm{Bc}, 82.45 . \mathrm{Mp}, 71.15 . \mathrm{Pd}, 82.65 .+\mathrm{r}$
\end{abstract}

Selective sieving of ions and molecules through thin membranes is a key step for a wide range of applications such as water purification and ion exchange membrane fuel cells [1-16]. Two-dimensional (2D) materials like graphene and hexagonal boron nitride (h-BN) offer potential as membrane materials since they are a single atom thick and have high mechanical stability and flexibility $[1,2,4,5,7-10]$. For some time, it was believed that pristine graphene and h-BN were impermeable to ions due to high energy barriers for penetration $[17,18]$. Recent experiments, however, have suggested that protons can in fact penetrate pristine graphene and h-BN $[1,2]$. In the measurements, the $2 \mathrm{D}$ materials were immersed in proton conducting polymers or aqueous solutions, and from temperature $(T)$-dependent proton conductivity measurements, proton penetration barriers of only 0.8 and $0.3 \mathrm{eV}$ were estimated for single-layer graphene and h-BN, respectively [1]. Note that these estimated barriers include contributions from zero-point energy (ZPE) [2]. Defects such as atomic pinholes are known to facilitate proton transfer [9]. A certain level of defects will inevitably be present, associated e.g. with $\mathrm{sp}^{3}$ carbon atoms [19]. However in Refs. [1, 2], various measurement techniques (transmission/tunnelling electron microscopy, Raman spectroscopy and measurements of gas leakage) were used to support the assertion that the proton transfer mechanism was not facilitated by atomic defects in the membranes.

Considerable theoretical effort has been devoted towards understanding the microscopic details of how protons penetrate $2 \mathrm{D}$ materials $[9,17,18,20-22]$. It has been established on the basis of density-functional theory (DFT) calculations that the barriers to proton pen- etration through pristine graphene and h-BN in vacuum can be excessively high. Specifically, computed barriers of 3.5-4.0 eV have been reported for chemisorbed protons (i.e. protons that are covalently bonded to the $2 \mathrm{D}$ materials) to penetrate graphene $[17,18,23]$. If the protons do not chemisorb on the surface but rather penetrate the sheet via a metastable physisorption state, smaller barriers of 1.4-2.6 eV have been reported [17, 18, 23]. However, the physisorption state is only a very shallow minimum, separated from the much more stable chemisorption state by a barrier of $\leq 0.1 \mathrm{eV}[24]$. Therefore, it seems unlikely that penetration from the physisorption state is the dominant mechanism for fast proton conduction $[22,24]$. Nonetheless this indicates that hydrogenation of graphene is facile and that graphene sheets immersed in proton conducting polymers or aqueous solutions could be hydrogenated or protonated to some extent. In addition, given the light mass of the proton, the role of nuclear quantum effects (NQEs) such as tunnelling and zero point motion could be relevant to the process, as shown e.g. through two interesting recent computational studies [21, 23].

In this Letter, we report a study of proton transfer through graphene and h-BN, focusing on the transmission mechanism. Consistent with earlier studies, a very high potential energy barrier of $\sim 3.6 \mathrm{eV}$ is found for proton penetration of graphene via the chemisorption state. Using ab initio path-integral molecular dynamics (PIMD) [25-31], we take into account nuclear quantum effects (NQEs) and finite temperature thermal effects. We find that NQEs reduce the penetration barrier of graphene by $0.46 \mathrm{eV}(12 \%)$ at $300 \mathrm{~K}$, which is unlikely to be responsible for the experimentally observed 
high transfer rate. Upon considering the role $\mathrm{sp}^{3}$ bonded atoms play on the penetration process, created here by hydrogenation of graphene and h-BN, we find that hydrogenation can reduce the penetration barriers significantly to less than $1 \mathrm{eV}$. This reduction arises because the hydrogenation induced $\mathrm{sp}^{2}$ to $\mathrm{sp}^{3}$ transformation destabilises the deep-lying chemisorption state in which the proton can get trapped on the pristine membranes. Geometrically, hydrogenation also expands the six-atom rings through which protons transfer. Analysis of the penetration barriers associated with many distinct hydrogenated membranes reveals a clear correlation between the height of the penetration barriers and the local degree of hydrogenation at the proton transfer site. Overall this work highlights the significant difference in proton penetration barriers that can be found in the vicinities of $\mathrm{sp}^{3}$ bonded atoms and helps to rationalise the facile transport of protons through single-layer materials.

Our DFT calculations were performed using the Vienna ab initio Simulation Package (VASP) [32], with an in house implementation of the $a b$ initio constrainedcentroid MD/PIMD methods [28, 33]. The optB88-vdW functional was chosen in the electronic structure calculations so as to obtain a good description of the hydrogen (H)-bonding interactions and dispersion forces [34, 35]. Charged cells were employed to describe the protons in the simulations, and we confirmed that any charge states considered were correctly characterized with Bader analysis $[36,37]$. We hydrogenated graphene to varying degrees without generating pinholes, using supercells ranging from $4 \times 4$ to $8 \times 8$. After hydrogenation, the supercell shape and size was allowed to change. For each partially hydrogenated structure, we have considered the two lowest energy structures following the study of Ref. [38]. The climbing image nudged elastic band (cNEB) method was used in calculating the static penetration barriers [39], with a force convergence criterion of $0.03 \mathrm{eV} \AA^{-1}$ and all atoms were allowed to relaxed. Beyond the static description, the classical and quantum free energy profiles were obtained with constrained MD and PIMD approaches [29-31]; with the constraint applied on the vertical distance of the proton from the 2D layer. A 0.5 fs time step was used and the imaginary-time path in the PIMD simulations was sampled with 48 replicas, at a target temperature of $300 \mathrm{~K}$. After thermalization, 30,000 steps (15 ps) were collected to calculate the constraint force, for each constraint point. By integrating over the constraint forces, the free energy profiles were obtained as detailed in the supplementary information (SI).

On free-standing graphene protons adsorb preferentially at the chemisorption site directly above a carbon atom (Fig. 1 (a)). From the chemisorption site, our calculations yield a proton penetration barrier of $3.60 \mathrm{eV}$. As noted, in previous experiments, the 2D layers were surrounded by proton conducting polymers or aqueous solutions $[1,2,9,20,22]$. In the current study, we do not aim to model such an aqueous environment. However, in order to gain an initial understanding of how the presence of water might impact upon the proton penetration process, we employed the simplified model shown in the inset of Fig. 1 (b). This model contains one water molecule on each side of the graphene layer and with the addition of a proton it enables us to model proton transfer from an $\mathrm{H}_{3} \mathrm{O}^{+}$on one side of the sheet to an $\mathrm{H}_{2} \mathrm{O}$ on the other side of the sheet [40]. Our calculations show that the proton adsorbs at either the water molecule or the chemisorption site of the graphene sheet with very similar stability (Fig. 1 (b)). The metastable physisorption site for protons on free-standing graphene, as illustrated in Fig. 1 (a), disappears due to the presence of water. The energy barrier for a proton to transfer from the $\mathrm{H}_{3} \mathrm{O}^{+}$to the chemisorption site is less than $0.1 \mathrm{eV}$. The penetration barrier from the chemisorption site is $3.65 \mathrm{eV}$ when water is present, very similar to the $3.60 \mathrm{eV}$ obtained in the absence of water. The energy differences between physisorbed water molecules on different sites or with different orientations are only a few meV [4042], so different configurations of water molecules will not obviously influence the energy profile of the proton penetration process. Therefore, in agreement with recent work [22], we conclude that the presence of water molecules is unlikely to change the fact that very high barriers exist for protons to transfer across the graphene layer.
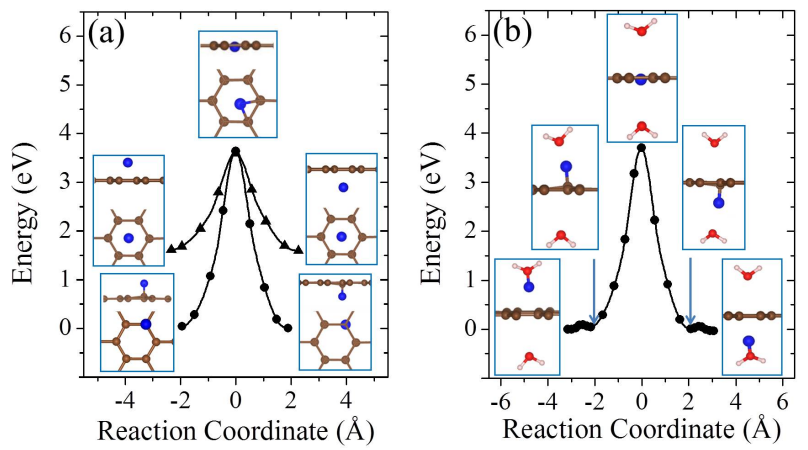

FIG. 1: High barriers for proton transfer through pristine graphene. Calculated energy profiles as obtained from cNEB calculations, for proton transfer across (a) pristine graphene, and (b) graphene with adsorbed water molecules. Two energy profiles are shown in (a), one between the metastable physisorption states (upper curve) and one between the chemisorption states (lower curve). The insets show some of the key states involved in the proton transfer processes. Brown (red, pink) balls are $\mathrm{C}(\mathrm{O}, \mathrm{H})$ atoms. Protons are represented by blue balls.

Finite temperature and NQEs [ZPE and quantum tunneling] are known to alter the barriers of chemical processes, particularly proton transfer barriers. To under- 
stand the importance of such effects on the current system, we performed a series of $a b$ initio MD and PIMD simulations from which free energy barriers for proton penetration were obtained. The results of these simulations are shown in Fig. 2. We find that the pure thermal effects on the barrier are relatively small and the free energy barrier in the model containing a water on either side of the sheet is $3.70 \mathrm{eV}$ at $300 \mathrm{~K}$. When NQEs are accounted for with PIMD the barrier is reduced by $0.46 \mathrm{eV}$ at $300 \mathrm{~K}$ (Fig. 2), quite a substantial reduction. Analysis reveals that this reduction in the free energy barrier is due to enhanced quantum delocalisation of the proton at the transition state compared to the initial state. This is similar behavior to that observed for $\mathrm{H}$ chemisorption on graphene [24], and is illustrated by the snapshots shown in Fig. 2. The reduction arising from NQEs is also in line with that reported by Poltavsky et al. when similar PIMD methods are used [23], although a different computational model and reaction pathway was considered by Poltavsky et al.. However, considering the fact that the free energy barrier for the process examined remains $>3 \mathrm{eV}$ at $300 \mathrm{~K}$, we conclude that NQEs alone can't rationalise the experimentally observed fast proton transfer.

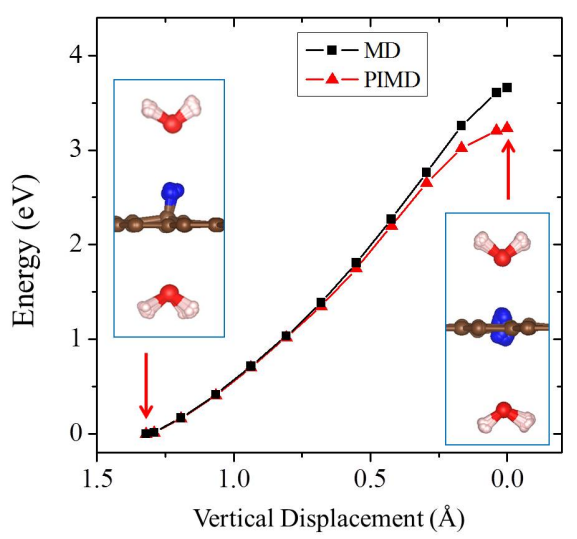

FIG. 2: Free energy profiles at $300 \mathrm{~K}$ obtained with $a b$ initio constrained MD and PIMD simulations for proton transfer across a graphene sheet in the presence of water molecules. The MD simulations take into account thermal effects, whereas the PIMD simulations capture thermal and nuclear quantum effects. PIMD simulation snapshots for the initial state and transition state are also shown. Blue (red and pink) balls represent the beads of protons ( $\mathrm{O}$ and $\mathrm{H}$ atoms), for one snapshot in a PIMD simulation. The centroids of the $\mathrm{C}$ atoms are shown as brown balls.

We noted in the introduction that carbon atoms with $\mathrm{sp}^{3}$ character are invariably present even in pristine graphene [19]. With this in mind we explored how the presence of chemisorbed hydrogens impact the proton penetration barrier of graphene. Adsorbed hydrogens are examined since when they chemisorb they lead to an $\mathrm{sp}^{2}$ to $\mathrm{sp}^{3}$ hybridisation of the carbon atoms they are bonded to but also because the hydrogenation of graphene is facile [43]. A broad range of hydrogenation scenarios was considered ranging from having just a single chemisorbed hydrogen at a proton penetration site to fully hydrogenated graphene (graphane) sheets. Examples of some of the structures considered are shown in Fig. 3, with full details given in the SI [44]. Upon computing the proton penetration barriers through the various hydrogenated and partially hydrogenated sheets considered, we find that hydrogenation leads to reduced proton penetration barriers. The actual barriers obtained depends sensitively on the particular hydrogenation structure, with barriers for some hydrogenated structures reduced very substantially to $<1 \mathrm{eV}$.
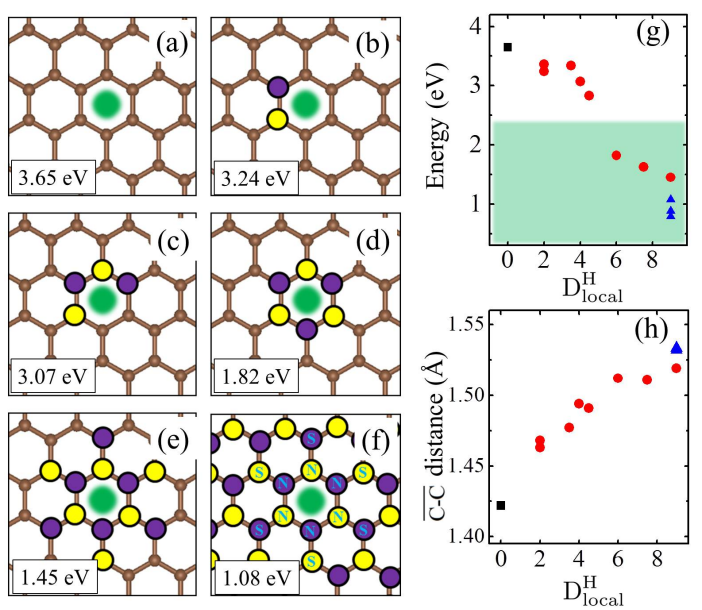

FIG. 3: Hydrogenation facilitates proton penetration through graphene. (a)-(f) The atomic structures of graphene and a selection of hydrogenated graphene sheets with different degrees of local hydrogenation. cNEB energy barriers for proton transfer through each sheet are also reported. Yellow and violet dots indicate $\mathrm{C}$ atoms hydrogenated from the top and bottom sides, respectively. The large smeared green ball indicates the hole of the $\mathrm{C}$ ring through which the proton penetration. In (f) the nearest neighbor $(\mathrm{N})$ and second nearest neighbor (S) C atoms to the penetration site are indicated. (g) cNEB barriers as a function of the degree of local hydrogenation $\left(\mathrm{D}_{\text {local }}^{\mathrm{H}}\right)$, for various hydrogenated structures(see Figs. S4 and S5 for details of the structures). The green shaded area indicates low penetration barriers with high $\mathrm{D}_{\text {local }}^{\mathrm{H}}$. (h) The averaged $\mathrm{C}-\mathrm{C}$ bond distance of the six- $\mathrm{C}$ ring through which the proton penetrates as a function of $\mathrm{D}_{\text {local }}^{\mathrm{H}}$. In $(\mathrm{g})$ and $(\mathrm{h})$, the black square, red dots and blue triangles represent data for proton transfer across pristine, partially hydrogenated and fully hydrogenated graphene, respectively.

Let us now look more closely at the hydrogenated systems and try to understand the barriers obtained. Crucially we find that the penetration of protons through a single atom layer is a local process and that the height of the penetration barrier depends primarily on the local degree of hydrogenation in the vicinity of the penetration 
site. To show this more clearly, we introduce an order parameter, $\mathrm{D}_{\text {local }}^{\mathrm{H}}$. $\mathrm{D}_{\text {local }}^{\mathrm{H}}$ is defined as $\mathrm{D}_{\text {local }}^{\mathrm{H}}=\mathrm{N}_{\mathrm{N}}^{\mathrm{H}}+\mathrm{w} \times \mathrm{N}_{\mathrm{S}}^{\mathrm{H}}$, with $\mathrm{N}_{\mathrm{N}}^{\mathrm{H}}\left(\mathrm{N}_{\mathrm{S}}^{\mathrm{H}}\right)$ being the number of hydrogenated atoms at the nearest (second nearest) neighbors of the hole (indicated by $\mathrm{N}$ and $\mathrm{S}$ in the Fig. 3 (f)), and w representing a weight factor capturing the importance of hydrogenation at the second nearest sites. The barriers as a function of $\mathrm{D}_{\text {local }}^{\mathrm{H}}$, with w set to 0.5 , are plotted in Fig. 3 (g) (tuning $\mathrm{w}$ from 0.2 to 0.8 gives similar results (Fig. S11)). Upon computing $\mathrm{D}_{\text {local }}^{\mathrm{H}}$ for all barriers considered we found two interesting features: i) a clear correlation exists between the penetration barrier and $\mathrm{D}_{\text {local }}^{\mathrm{H}}$, with the barrier getting smaller as $\mathrm{D}_{\text {local }}^{\mathrm{H}}$ inceases, and ii) the systems can be categorized into two main groups, with the most significant barrier reduction being found for $\mathrm{D}_{\text {local }}^{\mathrm{H}}>6$. Systems with $\mathrm{D}_{\text {local }}^{\mathrm{H}}$ smaller than 6 belong to the group with large barriers. In these systems, the six-C ring through which the proton penetrates is not fully hydrogenated. C atoms with $\mathrm{sp}^{2}$ bonding are present in the ring and the proton can chemisorb at these sites before penetration (Figs. S4 and S5). It is the presence of the very stable chemisorption sites that lead to particularly high barriers for proton penetration. For the systems considered, when $\mathrm{D}_{\text {local }}^{\mathrm{H}} \geq 6$, the ring is fully hydrogenated. The $\mathrm{sp}^{3}$ bonding eliminates the deep-lying chemisorption state before penetration. In so doing the initial state energy is raised and the barriers are lower than $2.0 \mathrm{eV}$. Note that this analysis reveals that because the barrier is related to the local extent of hydrogenation, a sample does not need to have a very high global degree of hydrogenation for low barrier proton penetration sites to exist. All that is required is a high local degree of hydrogenation and indeed surface science measurements and previous calculations show that upon hydrogenation there is a tendency for Hs to cluster $[38,45,46]$. Aside from eliminating the chemisorption well, $\mathrm{sp}^{3}$ bonded carbons also lead to an expansion of the lattice. This can be seen in Fig. 3 (h) where the averaged $\mathrm{C}-\mathrm{C}$ distance of a hexagon is shown to increase with $\mathrm{D}_{\text {local }}^{\mathrm{H}}$. This expansion is an additional geometric effect played by $\mathrm{sp}^{3}$ bonded carbons [47].

Our simulations with full hydrogenation correspond to the case when the graphene layer is fully hydrogenated around a local penetration site. They have the same $\mathrm{D}_{\text {local }}^{\mathrm{H}}$ but different barriers in Fig. 3 (g) (three blue triangles). To understand why this happens, we take the chair conformation and a disordered $\mathrm{H}$ configuration as examples and show the actual cNEB barrier profiles in Fig. 4. The key difference between these two systems is that in the chair conformation, the upper and lower sides of the graphene sheet are similarly hydrogenated, while in the disordered configuration the two sides of the sheet are hydrogenated to different extents. Such asymmetric decoration creates structures wherein it is yet more facile for the proton to penetrate form one side to the other. On a larger scale, one can imagine that hydrogenation can induce different local penetration sites, with the ease of penetration related to the extent of hydrogenation on either side of the sheet.

For h-BN, $\mathrm{H}$ atoms also prefer to chemisorb in pairs on $\mathrm{B}$ and $\mathrm{N}$ atoms, and the averaged binding energy between $\mathrm{H}$ atoms and h-BN increases with the degree of hydrogenation $[48,49]$. As with graphene, upon examining proton penetration through h-BN we find that the barriers decrease upon hydrogenation. As shown in Table I, the barrier through pristine h-BN is as high as 3.33 $\mathrm{eV}$. In fully hydrogenated h-BN with ordered $\mathrm{H}$ configurations (h-BN sheets with stirrup and boat conformations, h-BN $\mathrm{B}_{\text {stirrup-H }}$ and h- $\mathrm{BN}_{\text {boat-H }}$ ) [50], the barrier can be reduced to less than $2.0 \mathrm{eV}$. For disordered $\mathrm{H}$ configurations, the barrier further decreases to $\sim 0.93 \mathrm{eV}$. Some representative energy profiles for partially and fully hydrogenated h-BN sheets are provided in the Figs. S14S16.

In Table I, we summarize some representative barriers obtained for proton transfer through the various graphene and h-BN systems considered. Also included in Table I are the ZPE corrections to the barriers computed within the harmonic approximation. ZPE effects decrease the barriers to proton penetration in all systems considered and when they are taken into consideration the lowest barrier on graphene is $0.61 \mathrm{eV}$ and on h-BN it is $0.51 \mathrm{eV}$. Finally, we note that we have also considered how substitution of $\mathrm{H}$ for $\mathrm{D}$ is likely to alter the penetration barriers. Treating this again at the ZPE level we find a $50 \mathrm{meV}$ difference in penetration barriers between $\mathrm{H}$ and $\mathrm{D}$ for $\mathrm{G}_{\text {disordered-H}}$, and a $120 \mathrm{meV}$ difference between $\mathrm{H}$ and $\mathrm{D}$ for $\mathrm{h}-\mathrm{BN}_{\text {disordered-H. }}$. In each case, the $\mathrm{D}$ barrier is slightly larger than the $\mathrm{H}$ barrier, in agreement with recent computational work [21] and experiment [2].
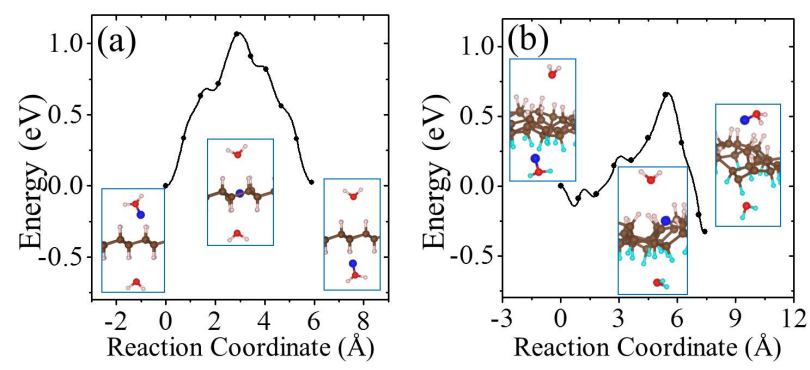

FIG. 4: Energy profiles for proton transfer across fully hydrogenated graphene with (a) the chair conformation and (b) a disordered $\mathrm{H}$ configuration. Insets show the atomic structures for the initial, transition and final states. Red (pink, brown) balls are $\mathrm{O}(\mathrm{H}, \mathrm{C})$ atoms. Protons are represented by blue balls. For the disordered conformation, the $\mathrm{H}$ adatoms below the sheets are colored with cyan (a contrast to pink) for clarity. The specific disordered and asymmetric decoration pattern reported in (b) yields a particularly low proton penetration barrier. Additional information on these structures is given in Fig. S13. 
To conclude, we have reported a theoretical study on proton transfer through graphene and h-BN. After considering various factors that could impact on the penetration barriers for protons, we find that $\mathrm{sp}^{3}$ hybridization at the penetration site, achieved here through hydrogenation, plays a key role in reducing these barriers to less than $1.0 \mathrm{eV}$. The physical origin of the barrier reduction is the elimination of the deep-lying chemisorption states and the expansion of the honeycomb lattice at the penetration site. Combining the major influence from hydrogenation and minor influence from NQEs, the experimentally observed low proton transfer barrier can be rationalised. Considering the fact that $2 \mathrm{D}$ materials can be functionalized with various elements other than $\mathrm{H}$, e.g. $\mathrm{O}, \mathrm{OH}, \mathrm{F}, \mathrm{Cl}$, this study suggests that there could be further scope for more controllable ion and proton sieving. We hope our study can stimulate further theoretical and experimental investigations in this direction.

Y.X.F., X.Z.L. and E.W. are supported by the National Basic Research Programs of China under Grand Nos. 2016YFA0300900, 2013CB934600, the National Science Foundation of China under Grant Nos 11275008, 1142243, 11274012, 91021007, 11604092 and 11634001. J.C. and A.M. are supported by the European Research Council under the European Union's Seventh Framework Programme (FP/2007-2013)/ERC Grant Agreement number 616121 (HeteroIce project). A.M. is also supported by the Royal Society through a Royal Society Wolfson Research Merit Award. The computational resources were provided by the supercomputer TianHe-1A in Tianjin, China and ARCHER from the UKCP consortium (EP/F036884/1).

\begin{tabular}{|c|c|c|c|}
\hline & cNEB Barrier & $\triangle E_{\mathrm{ZPE}}$ & Barrier \\
\hline $\mathrm{G}_{\text {pristine }}$ & 3.65 & -0.26 & 3.39 \\
\hline $\mathrm{G}_{\text {chair-H }}$ & 1.08 & -0.07 & 1.06 \\
\hline $\mathrm{G}_{\text {boat-H }}$ & 0.88 & -0.12 & 0.76 \\
\hline $\mathrm{G}_{\text {disordered-H }}$ & 0.79 & -0.18 & 0.61 \\
\hline h-BN $\mathrm{BN}_{\text {pristine }}$ & 3.33 & -0.21 & 3.12 \\
\hline h-BN stirrup-H $_{\text {in }}$ & 1.43 & -0.53 & 0.90 \\
\hline h-BN $\mathrm{Boat}_{\text {bot }}$ & 1.91 & -0.35 & 1.56 \\
\hline h-BN $\mathrm{BN}_{\text {disordered-H }}$ & 0.93 & -0.39 & 0.51 \\
\hline
\end{tabular}

TABLE I: Calculated cNEB barrier, ZPE corrections $\left(\triangle E_{\mathrm{ZPE}}\right)$ and corrected barrier (Barrier) for proton transfer across pristine and hydrogenated graphene and h-BN sheets. The rightmost column should be compared with the experimental values of $0.8 \mathrm{eV}$ and $0.3 \mathrm{eV}$ in Ref. 1 . $\triangle E_{\mathrm{ZPE}}$ is estimated as the ZPE differences between the initial and transition states. $\mathrm{G}_{\text {pristine }}$ and $\mathrm{h}-\mathrm{BN}_{\text {pristine }}$ are pristine graphene and h-BN sheets. $\mathrm{G}_{\text {chair-H }}, \mathrm{G}_{\text {boat-H}}, \mathrm{G}_{\text {disordered-H}}$, $\mathrm{h}-\mathrm{BN}_{\text {stirrup-H}}, \mathrm{h}-\mathrm{BN}_{\text {boat-H }}$ and $\mathrm{h}-\mathrm{BN}_{\text {disordered-H }}$ are hydrogenated $2 \mathrm{D}$ sheets with various $\mathrm{H}$ conformations. Water molecules are present on either side of the sheet for all systems reported here. The lowest barrier for each material, is indicated in bold.
* These two authors contributed equally

$\dagger$ Electronic address: angelos.michaelides@ucl.ac.uk

¥ Electronic address: xzli@pku.edu.cn

[1] S. Hu et al., Nature 516, 227 (2014).

[2] M. Lozada-Hidalgo et al., Science 351, 68 (2016).

[3] J. Abraham et al., Nat. Nanotech. (2017).

[4] S. P. Surwade et al., Nat. Nanotech. 10, 459 (2015).

[5] D. Cohen-Tanugi and J. C. Grossman, Nano Lett. 12, 3602 (2012).

[6] A. Zilman, J. Pearson, and G. Bel, Phys. Rev. Lett. 103, 128103 (2009).

[7] S. C. O'Hern et al., ACS Nano 6, 10130 (2012).

[8] K. Celebi et al., Science 344, 289 (2014).

[9] J. L. Achtyl et al., Nat. Commun. 6, 6539 (2015).

[10] S. C. O'Hern et al., Nano Lett. 14, 1234 (2014).

[11] R. Joshi et al., Science 343, 752 (2014).

[12] B. Mi, Science 343, 740 (2014).

[13] H. W. Kim et al., Science 342, 91 (2013).

[14] H. Li, J. S. Francisco, and X. C. Zeng, Proc. Natl. Acad. Sci. 112, 10851 (2015).

[15] C. Cheng et al., Sci. Adv. 2, e1501272 (2016).

[16] E. Secchi et al., Nature 537, 210 (2016).

[17] M. Miao, M. B. Nardelli, Q. Wang, and Y. Liu, Phys. Chem. Chem. Phys. 15, 16132 (2013).

[18] W. L. Wang and E. Kaxiras, New J. Phys. 12, 125012 (2010).

[19] Z. H. Ni et al., Nano Lett. 10, 3868 (2010).

[20] M. I. Walker et al., Appl. Phys. Lett. 107, 213104 (2015).

[21] Q. Zhang, M. Ju, L. Chen, and X. C. Zeng, J. Phys. Chem. Lett. 7, 3395 (2016).

[22] J. M. H. Kroes, A. Kroes, and M. I. Katsnelson, Phys. Chem. Chem. Phys. 19, 5813 (2017).

[23] I. Poltavsky, L. Zheng, M. Mortazavi, and A. Tkatchenko, arXiv 1605.06341v1 (2016).

[24] E. R. M. Davidson, J. Klimeš, D. Alfè, and A. Michaelides, Nano Lett. 8, 9905 (2014).

[25] D. Marx and M. Parrinello, Z. Phys. B: Condens. Matter 95, 143 (1994).

[26] M. E. Tuckerman, D. Marx, M. L. Klein, and M. Parrinello, J. Chem. Phys. 104, 5579 (1996).

[27] M. E. Tuckerman, D. Marx, M. L. Klein, and M. Parrinello, J. Chem. Phys. 275, 817 (1997).

[28] J. Guo et al., Science 352, 321 (2016).

[29] M. E. Tuckerman and D. Marx, Phys. Rev. Lett. 86, 4946 (2001).

[30] Q. Zhang et al., Phys. Rev. Lett. 101, 215902 (2008).

[31] B. Walker and A. Michaelides, J. Chem. Phys. 133, 174306 (2010).

[32] G. Kresse and J. Furthmüller, Comput. Mat. Sci 6, 15 (1996).

[33] J. Chen et al., Nat. Commun. 4, 2064 (2013).

[34] J. Klimeš, D. R. Bowler, and A. Michaelides, J. Phys.: Condens. Matter 22, 022201 (2010).

[35] J. Klimeš, D. R. Bowler, and A. Michaelides, Phys. Rev. B 83, 195133 (2011).

[36] R. Bader, Atoms in Molecules: A Quantum Theory, (Oxford University Press, New York, 1990).

[37] G. Henkelman, A. Arnaldsson, and H. Jónsson, Comput. Mater. Sci. 36, 254 (2006).

[38] Z. Šljivančanin, M. Andersen, L. Hornekær, and B. Hammer, Phys. Rev. B 83, 205426 (2011). 
[39] G. Henkelman, B. P. Uberuaga, and H. Jónsson, J. Chem. Phys. 113, 9901 (2000).

[40] T. Kurita, S. Okada, and A. Oshiyama, Phys. Rev. B 75, 205424 (2007).

[41] J. Ma et al., Phys. Rev. B 84, 033402 (2011).

[42] T. Hamada, Phys. Rev. B 86, 195436 (2012).

[43] C. F. Lin et al., Nano Lett. 15, 903 (2015).

[44] See supplementary material at http://xxx for details of the method and computational setups.
[45] L. Hornekær et al., Phys. Rev. Lett. 97, 186102 (2006).

[46] N. P. Guisinger et al., Nano Lett. 9, 1462 (2009).

[47] O. Leenaerts et al., Phys. Rev. B 82, 195436 (2010).

[48] J. M. H. Kroes, A. Fasolino, and M. I. Katsnelson, Phys. Chem. Chem. Phys. 18, 19359 (2016).

[49] V. V. Khvostov et al., Appl. Surf. Sci. 157, 178 (2000).

[50] D. K. Samarakoon and X. Q. Wang, Appl. Phys. Lett. 100, 103107 (2012). 


\title{
Supplementary Information: Hydrogenation Facilitates Proton Transfer Through Two-Dimensional Honeycomb Crystals
}

\author{
Yexin Feng, ${ }^{1, *}$ Ji Chen, ${ }^{2, *}$ Wei Fang, ${ }^{3}$ Enge \\ Wang, ${ }^{4}$ Angelos Michaelides, ${ }^{2, \dagger}$ and Xin-Zheng $\mathrm{Li}^{4, \ddagger}$ \\ ${ }^{1}$ School of Physics and Electronics, \\ Hunan University, Changsha 410082, P. R. China \\ ${ }^{2}$ Thomas Young Centre, London Centre for Nanotechnology, \\ and Department of Physics and Astronomy, \\ University College London, London WC1E 6BT, United Kingdom \\ ${ }^{3}$ Thomas Young Centre, London Centre for Nanotechnology, \\ and Department of Chemistry, University College London, \\ London WC1E 6BT, United Kingdom \\ ${ }^{4}$ International Center for Quantum Materials, School of Physics, \\ and Collaborative Innovation Center of Quantum Matter, \\ Peking University, Beijing 100871, P. R. China
}

(Dated: September 29, 2018) 


\section{S.I COMPUTATIONAL DETAILS ABOUT DFT CALCULATIONS}

Spin-polarized density-functional theory (DFT) calculations were performed by using the Vienna ab initio Simulation Package (VASP) [1]. The projector-augmented plane wave (PAW) method was employed with a cut-off energy of $500 \mathrm{eV}$. The Monkhorst-Pack (MP) k-point meshes of $2 \times 2 \times 1$ and $1 \times 1 \times 1$ are used for supercells with the surface periodicity of $4 \times 4$ and $8 \times 8$. A slab layer of $15 \AA$ thick was enough to avoid interactions between the layers. We performed climbing image nudged elastic band (cNEB) claculations to get the proton penetration barriers across 2D materials [2], with a force convergence criterion of 0.03

$\mathrm{eV} \AA^{-1}$. The optB88-vdW functional was chosen for the electronic structure calculations so as to obtain a good description of the dispersion forces $[3,4]$. Here we check the influence of using other functionals on the calculated penetration barriers for proton. We consider two kinds of penetration processes for the proton, a high barrier process with pristine graphene and a low barrier process with hydrogenated graphene, as shown in Fig. S1. The cNEB barriers for proton penetration obtained with optB88-vdW [3, 4], LDA [5, 6], PBE [7], PBEsol [8], and PBE0 [9, 10] functionals ar summarized in Table SI. Similar cNEB barriers for proton penetration are obtained with various functionals.

\section{S.II HYDROGENATED GRAPHENE AND H-BN SHEETS}

In the main manuscript, we have discussed the influence of hydrogenation on the proton penetration through graphene and h-BN sheets. The atomic structures of fully hydrogenated graphene and h-BN sheets with ordered H-configurations are shown in FIG. S2. Apart from the ideally hydrogenated graphene sheets, we have also studied the disordered $\mathrm{H}$ configurations, as shown in FIG. S3. A larger $(8 \times 8)$ supercell was employed, which contains 256 atoms. All C, N and B atoms are decorated by $\mathrm{H}$ atoms. The hydrogenation sites were determined randomly on either side of the 2D membrane, and structures were fully optimized. Besides these, the proton transfer barriers were also calculated for various partially hydrogenated graphene sheets. For each kind of partially hydrogenated graphene, the two lowest energy structures are considered, as shown in FIGs. S4 and S5.

As mentioned in the main text, the asymmetric decoration of $\mathrm{H}$ on the opposite sides of $2 \mathrm{D}$ layers can further slightly reduce the proton transfer barrier. The zoom-in view of 
atomic structures for this asymmetric $\mathrm{H}$ decoration on hydrogenated graphene and h-BN with disordered $\mathrm{H}$ conformations can be found in FIG. S6.

\section{S.III PIMD SIMULATIONS}

In this study, we employ constrained PIMD simulations to obtain the free energy profiles for proton penetration across graphene and h-BN sheets, by constraining the vertical displacement of proton from the 2D layers. During the PIMD simulations, the centroids of six atoms in the hexagon through which proton penetrates are allowed to relaxed; whereas the centroids of other atoms in the 2D sheets are fixed to the positions in the pristine 2D layers. In PIMD simulations, the number of beads used to sample the imaginary-time path-integral is a very important parameter in descriptions of the NQEs. We have therefore calculated the mean constraint force, for the proton above the graphene layer with the vertical dis-

placement of $0.18 \AA$, by using $1,4,8,16,24,48$ and 64 beads. The Anderson thermostat was used to target the temperature at $300 \mathrm{~K}$. The convergence tests are shown in FIGs. S7 and S8. The differences between the mean constraint forces with 24, 48 and 64 beads are less than $50 \mathrm{meV}$. 48 beads were used to for the reported PIMD results.

In the main manuscript we have shown the impact of NQEs on the free energy profile for proton transfer across pristine graphene. NQEs reduce the transfer barrier by $0.46 \mathrm{eV}$. In FIG. S9, we also plot the classical and quantum free energy profiles for proton penetration through the h-BN sheet. The reduction of barrier due to NQEs is $0.28 \mathrm{eV}$.

\section{S.IV ISOTOPE EFFECT AND ZERO POINT ENERGY (ZPE) CORRECTION}

The profiles of the mean constraint forces, for the proton and deuteron to penetrate through pristine graphene, are illustrated in FIG. S10. By integrating over the constraint forces, the free energy profiles are obtained for $\mathrm{H}$ and $\mathrm{D}$, respectively. For this specific process, the isotope effect is about $134 \mathrm{meV}$.

In Table I of the manuscript, for various proton penetration processes, we investigate the impact of NQEs on the penetration barrier by doing ZPE corrections for the initial and final states. The zone centered (Gamma-point) frequencies $\omega_{i}$ are calculated with the finite displacement method. The ZPEs of the initial and the final states are evaluated by $\sum \frac{1}{2} \hbar \omega_{i}$ 
using all frequencies. $\triangle E_{\mathrm{ZPE}}$ is calculated as the ZPE difference between the initial and the transition states. We can find that for hydrogenated h-BN sheets $\triangle E_{\mathrm{ZPES}}$ are larger. This is related to the distinct atomic structures and vibrational frequencies of the initial states for hydrogenated h-BN sheets. On h-BN, although the deep-lying chemisorbed state is avoided upon hydrogenation, the elevated chemisorbed state is still a well-defined local minimum and we take it as the initial state for the calculation of the barriers. In Fig. S12, the calculated $\triangle E_{\mathrm{ZPES}}$ along with the stretching frequencies of $\mathrm{O}-\mathrm{H}^{+}$bonds and $\mathrm{C} / \mathrm{N}-\mathrm{H}^{+}$ bonds in the initial state of each system considered are shown. One difference between the proton penetration mechanism through hydrogenated h-BN and that through hydrogenated graphene is that with fully hydrogenated graphene proton passes through the $\mathrm{C}$ ring directly from $\mathrm{H}_{3} \mathrm{O}^{+}$, while with fully hydrogenated h-BN the positively charged proton first adsorbs on negatively charged $\mathrm{N}$ before passing through the sheet, as shown in Figs. S13-S16. As the stretching frequencies of $\mathrm{O}-\mathrm{H}^{+}$bonds in $\mathrm{H}_{3} \mathrm{O}^{+}$are smaller than those of $\mathrm{C}-\mathrm{H}^{+}$and $\mathrm{N}-\mathrm{H}^{+}$ bonds on 2D layers, $\triangle E_{\mathrm{ZPE}}$ 's are larger in hydrogenated h-BN systems, as shown in Fig. S12.

* These two authors contributed equally

$\dagger$ Electronic address: angelos.michaelides@ucl.ac.uk

$\ddagger$ Electronic address: xzli@pku.edu.cn

[1] G. Kresse and J. Furthmüller, Comput. Mat. Sci 6, 15 (1996).

[2] G. Henkelman, B. P. Uberuaga, and H. Jónsson, J. Chem. Phys. 113, 9901 (2000).

[3] J. Klimeš, D. R. Bowler, and A. Michaelides, J. Phys. Condens. Matter 22, 022201 (2010).

[4] J. Klimeš, D. R. Bowler, and A. Michaelides, Phys. Rev. B 83, 195133 (2011).

[5] D. M. Ceperley, and B. J. Alder, Phys. Rev. Lett. 45, 566 (1980).

[6] J. P. Perdew, and A. Zunger, Phys. Rev. B 23, 5048 (1981).

[7] J. P. Perdew, K. Burke, and M. Ernzerhof, Phys. Rev. Lett. 77, 3865 (1996).

[8] J. P. Perdew et al., Phys. Rev. Lett. 100, 136406 (2008).

[9] C. Adamo, M. Cossi, G. Scalmani, and V. Barone, Chem. Phys. Lett. 307, 265 (1999).

[10] J. P. Perdew, K. Burke, and M. Ernzerhof, Phys. Rev. Lett. 77, 3865 (1996).

[11] Z. Šljivančanin, M. Andersen, L. Hornekær, and B. Hammer, Phys. Rev. B 83, 205426 (2011).

[12] D. K. Samarakoon and X. Q. Wang, Appl. Phys. Lett. 100, 103107 (2012). 

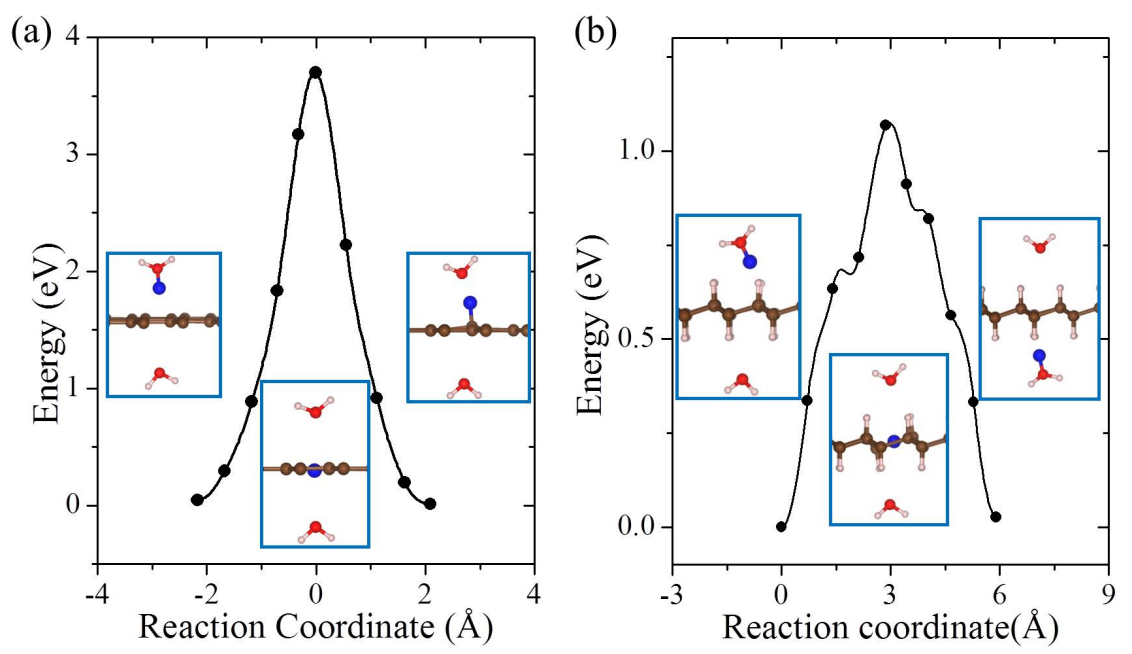

FIG. S1: (a) Energy profiles for proton penetration through pristine graphene. (b) Energy profiles for proton transfer across fully hydrogenated graphene with the chair conformation. Water molecules are present on either side of the sheet for the two systems considered here. The insets show the initial, transition and final states. Brown (red, pink) balls are $\mathrm{C}(\mathrm{O}, \mathrm{H})$ atoms. Protons are represented by blue balls.

\begin{tabular}{c|c|c}
\hline & $\begin{array}{c}\text { cNEB Barrier on } \\
\mathbf{G}_{\text {pristine }}(\mathbf{e V})\end{array}$ & $\begin{array}{c}\text { cNEB Barrier on } \\
\mathbf{G}_{\text {chair-H }}(\mathbf{e V})\end{array}$ \\
\hline LDA & 3.33 & 1.40 \\
\hline PBE & 3.57 & 1.11 \\
\hline PBEsol & 3.43 & 1.25 \\
\hline PBE0 & 3.58 & 1.17 \\
\hline optB88-vdW & 3.65 & 1.08 \\
\hline
\end{tabular}

TABLE SI: Calculated cNEB barriers for proton penetration through pristine graphene and fully hydrogenated graphene with the chair conformation, by using various functionals. 
(a)
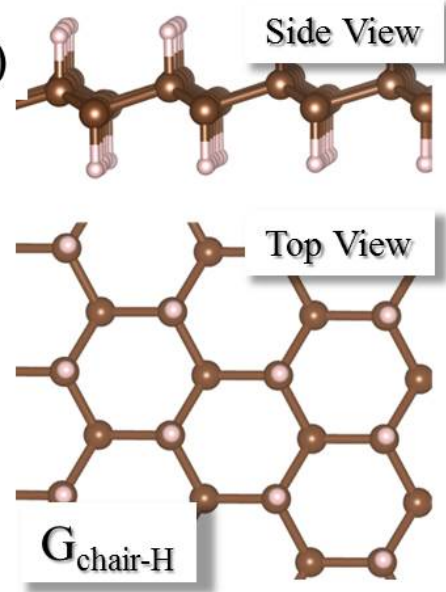

(c)
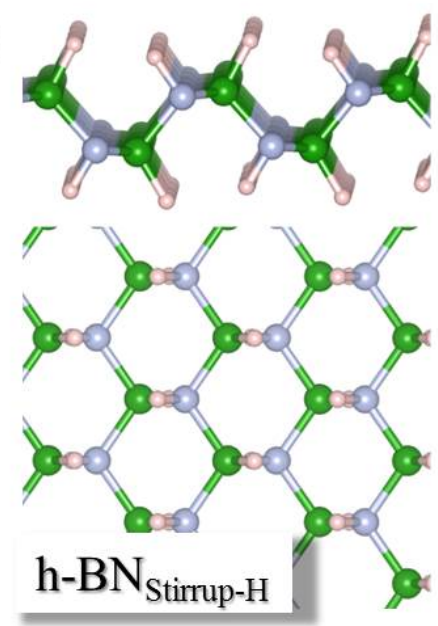

(b)
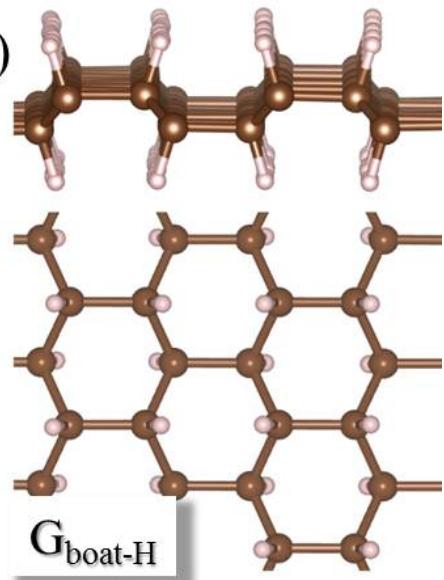

(d)
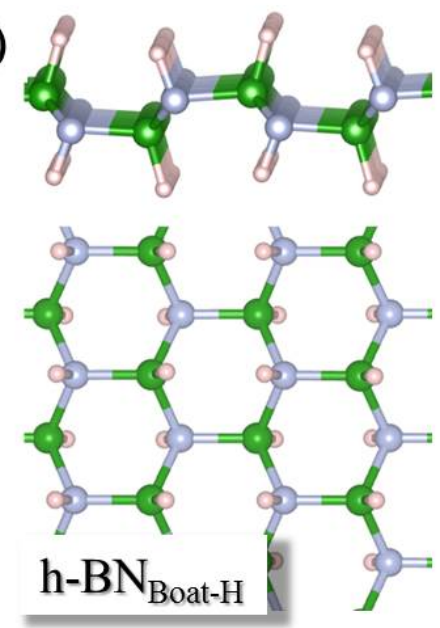

FIG. S2: Atomic structures of hydrogenated graphene with (a) the chair and (b) the boat conformations and hydrogenated h-BN with (c) the stirrup and (d) the boat conformations. Brown (pink, green, gray) balls are $\mathrm{C}(\mathrm{H}, \mathrm{B}, \mathrm{N})$ atoms $[11,12]$. 

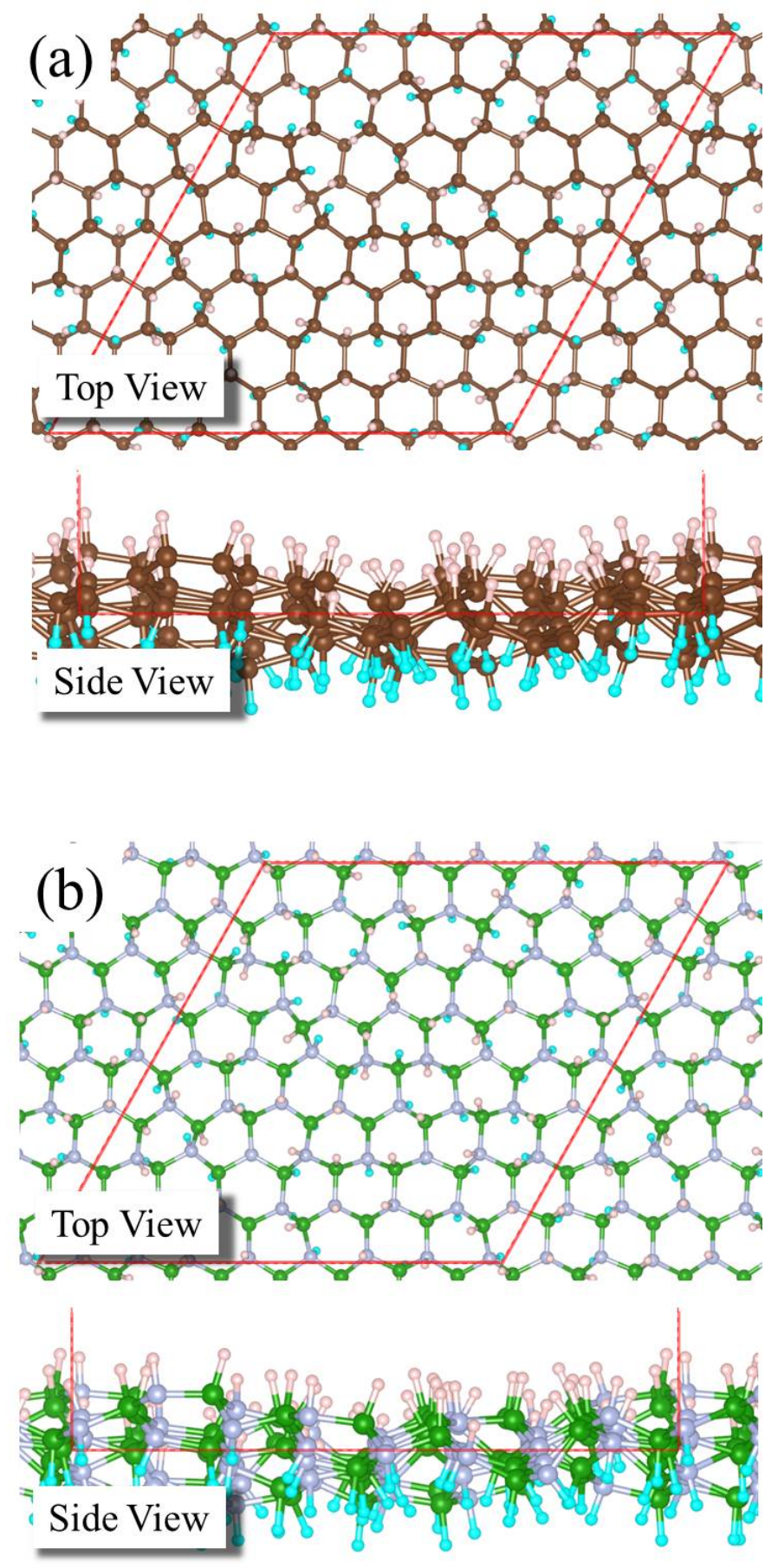

FIG. S3: Atomic structures of hydrogenated (a) graphene and (b) h-BN with disordered $\mathrm{H}$ configurations. Brown (pink/cyan, green, gray) balls are $\mathrm{C}(\mathrm{H}, \mathrm{B}, \mathrm{N})$ atoms. For easy visualization, the $\mathrm{H}$ adatoms below the sheets are colored in cyan. 

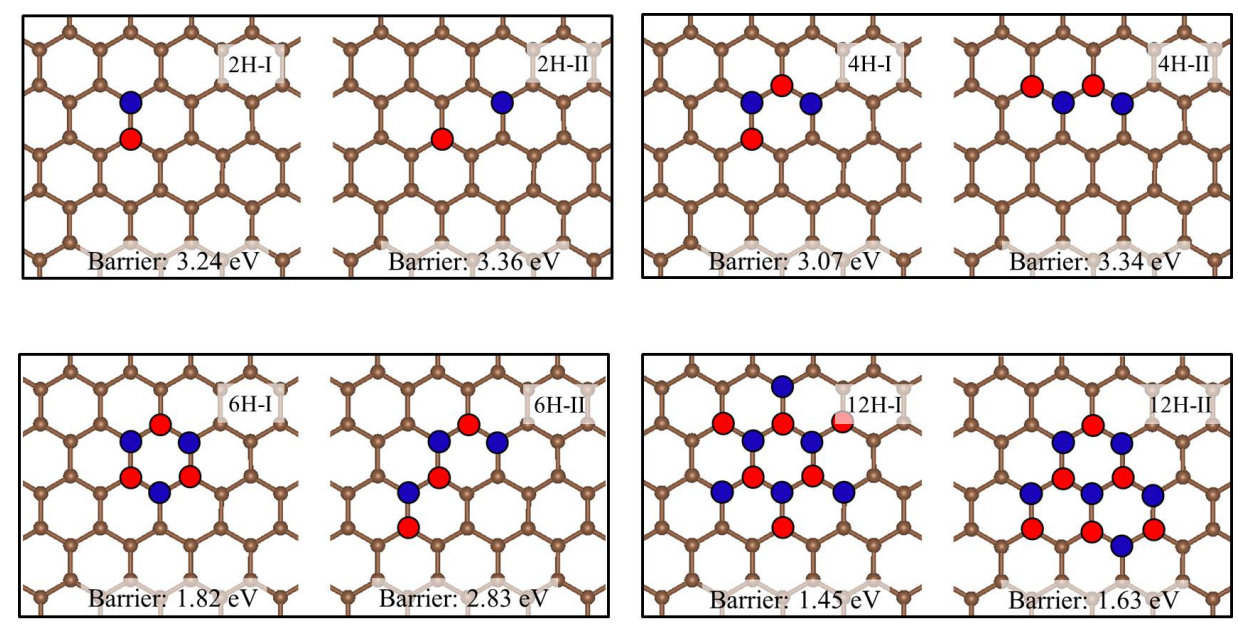

FIG. S4: The configurations of partially hydrogenated graphene with adsorbed H clusters on both sides of graphene. Red and blue dots indicate $\mathrm{C}$ atoms decorated by $\mathrm{H}$ atoms from the top and bottom side of graphene. The value of the cNEB barrier is also reported for each system.
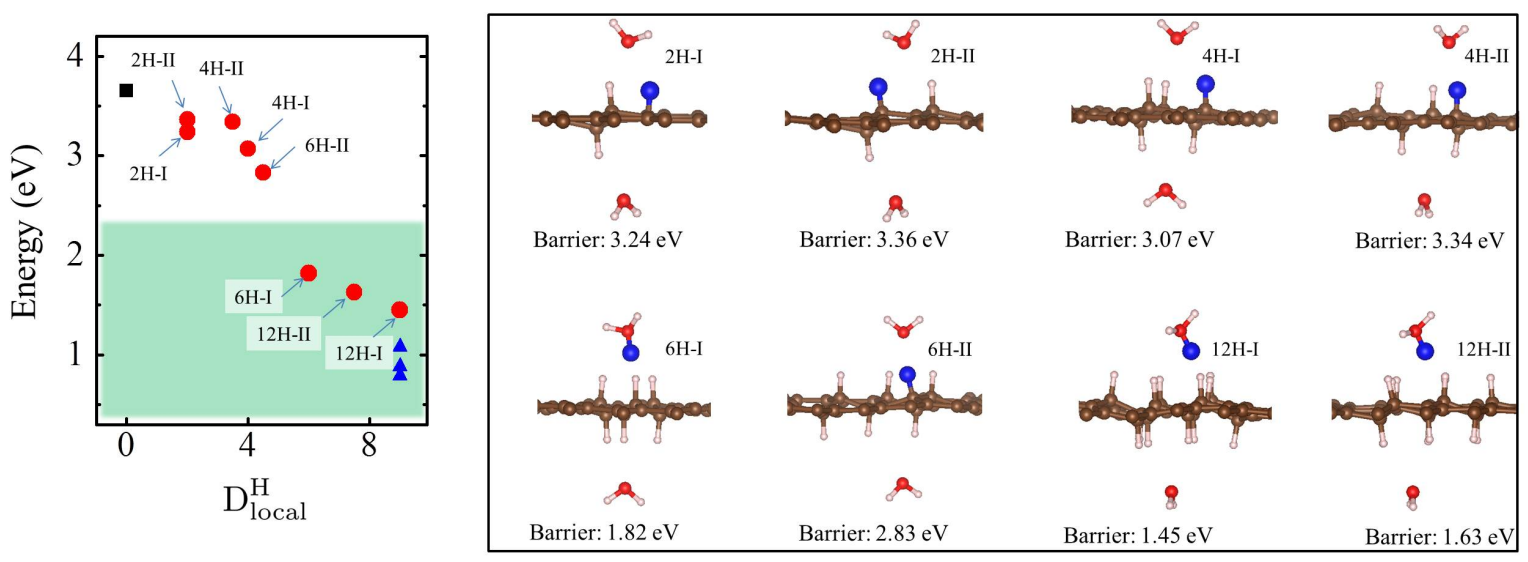

FIG. S5: cNEB barriers as a function of the local hydrogenation degree $\left(\mathrm{D}_{\text {local }}^{\mathrm{H}}\right)$ are shown in the left panel. The green shaded area indicates low penetration barriers with high $\mathrm{D}_{\text {local }}^{\mathrm{H}}$. In the right panel are the atomic structures of the initial states for the partially hydrogenated graphene as shown in the FIG S4. Red (pink, brown) balls are O (H, C) atoms. Protons are represented by blue balls. 


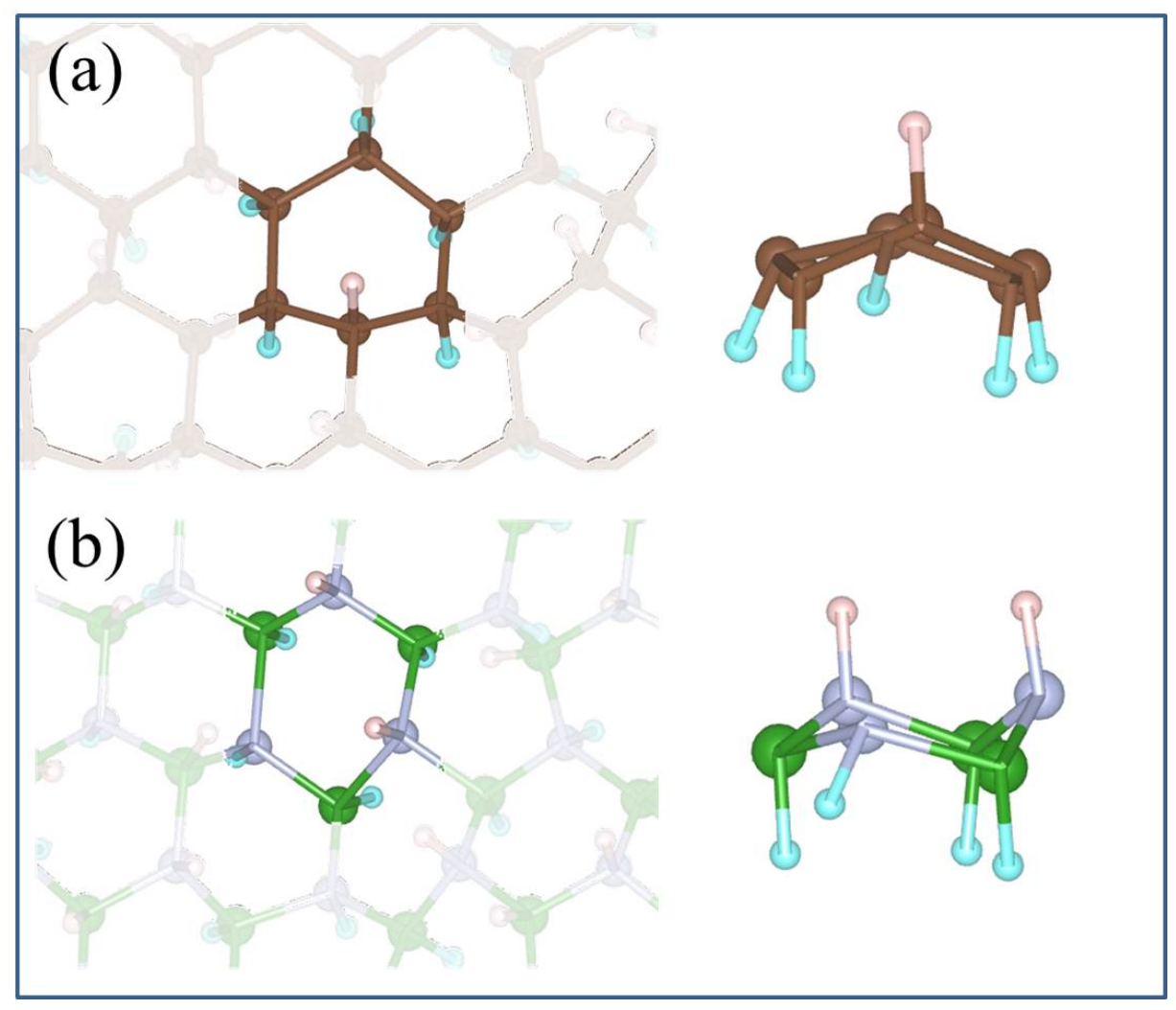

FIG. S6: The zoom-in view of atomic structures for asymmetric $\mathrm{H}$ decoration on hydrogenated (a) graphene and (b) h-BN with disordered H. Red (pink, brown, green, gray) balls are O (H, C, B, $\mathrm{N})$ atoms. The $\mathrm{H}$ adatoms below the sheets are colored with cyan (a contrast to pink) for clarity. The proton penetrates through the $2 \mathrm{D}$ material from the above to below of the sheet. The hexagon through which the proton penetrates is highlighted. 


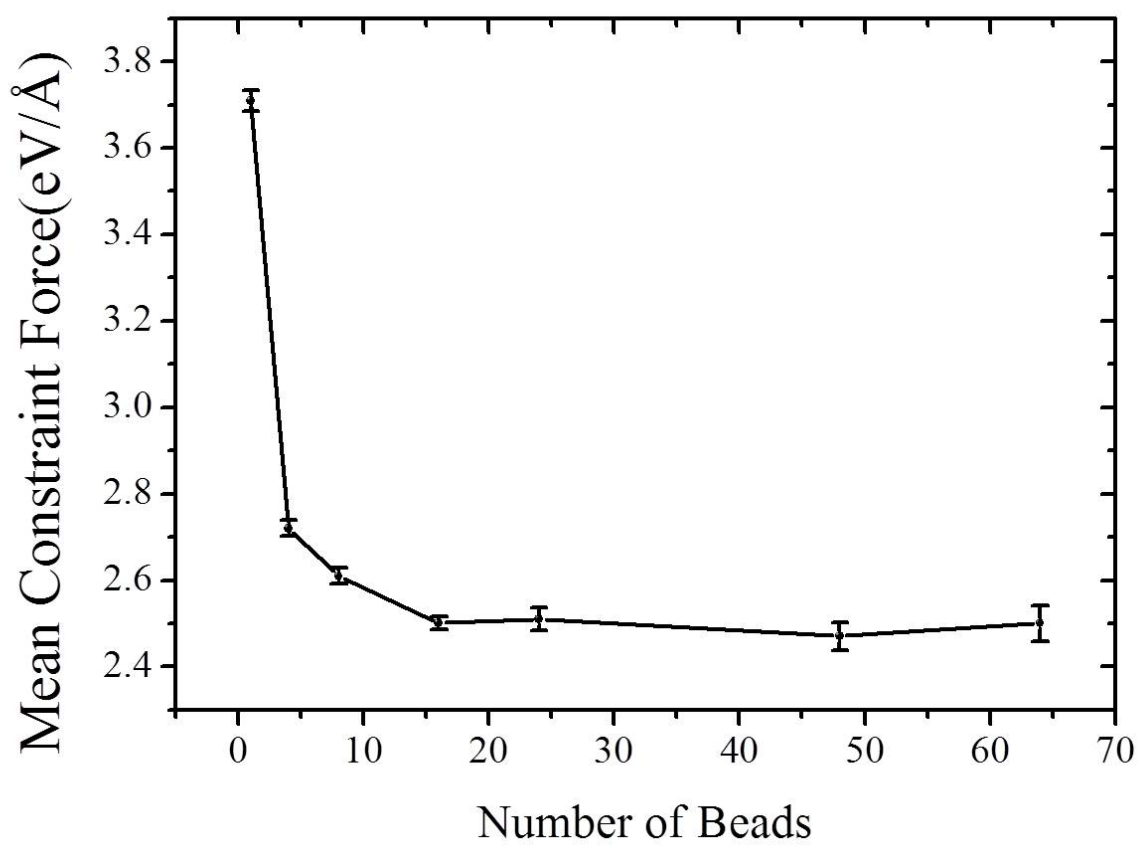

FIG. S7: The mean constraint force as a function of PIMD beads, for the proton above the graphene layer with a vertical displacement of $0.18 \AA$. A vertical displacement of 0 means the plane of the graphene layer (the $\mathrm{C}$ atoms fixed during the PIMD simulations). 

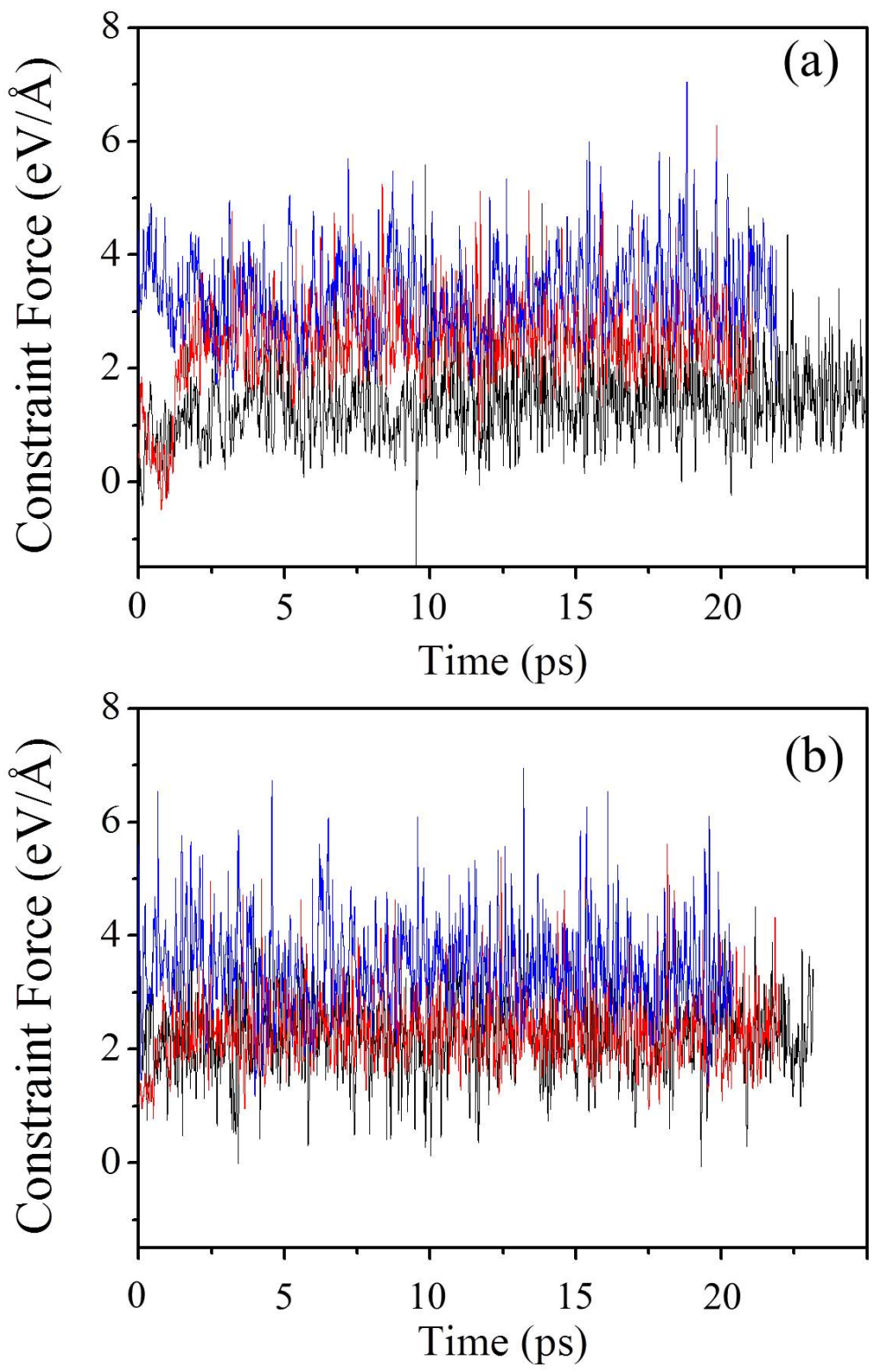

FIG. S8: The constraint forces during PIMD simulations, along with the simulation time for (a) $\mathrm{H}$ and (b) D nuclei, at the vertical displacement of $0.0389 \AA$ (black lines), $0.1671 \AA$ (red lines) and $0.2953 \AA$ (blue lines), respectively. After thermalization $(\sim 5 \mathrm{ps}), 30,000$ steps $(15 \mathrm{ps})$ were collected to calculate the constraint force, at each constraint point. 


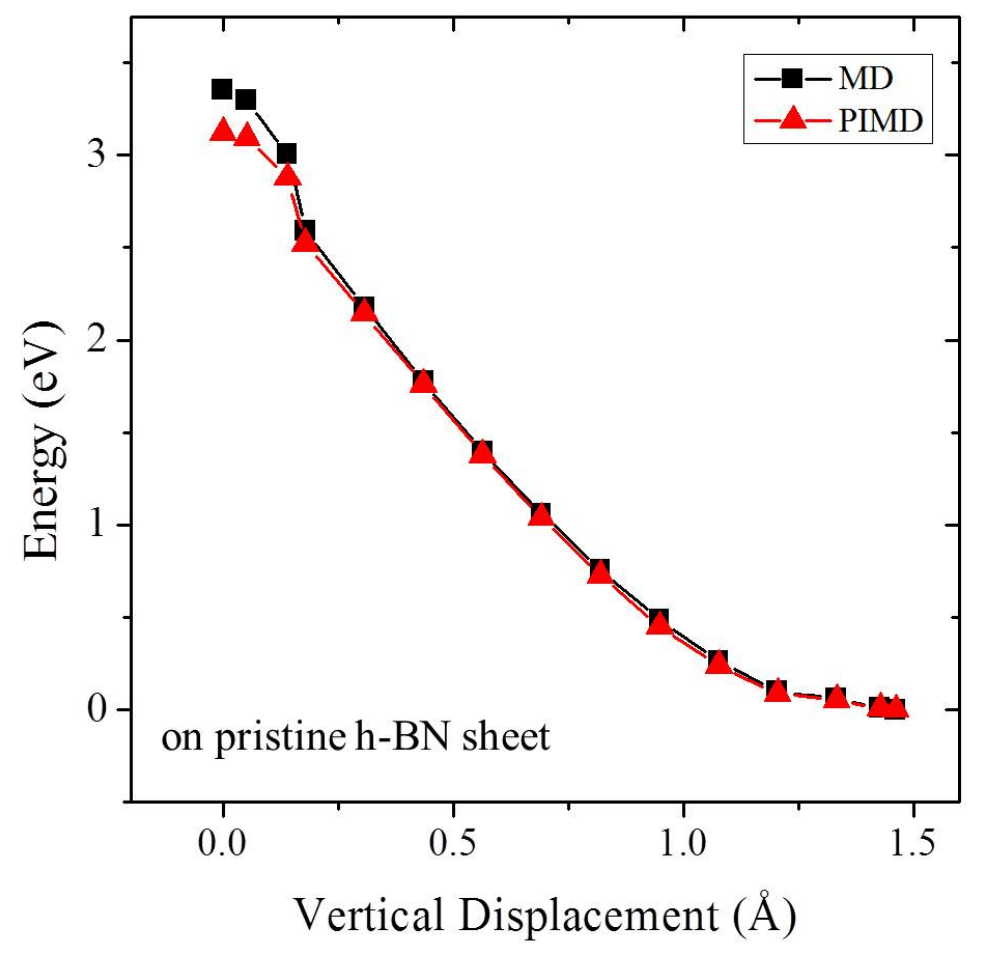

FIG. S9: The classical and quantum free energy profiles for proton transfer across single-layer pristine h-BN sheet, based on constrained MD/PIMD simulations. 


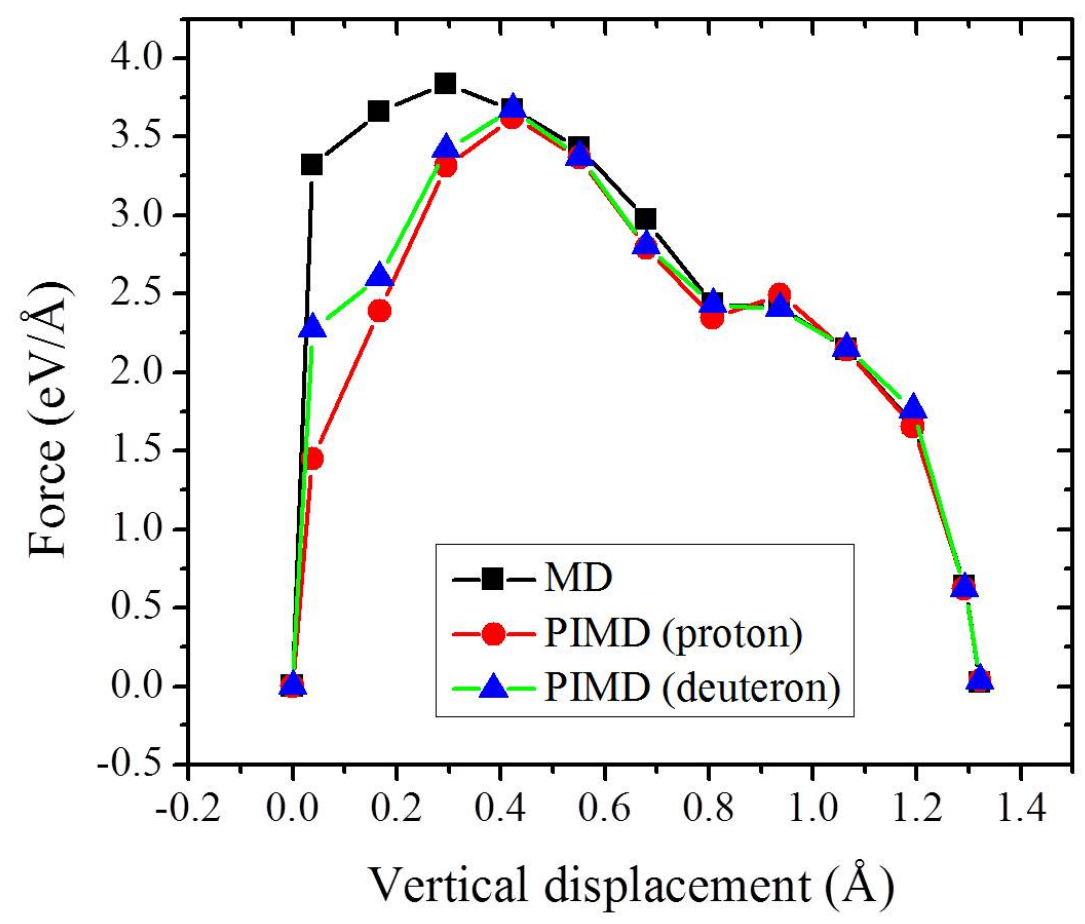

FIG. S10: The classical and quantum (proton/deuteron) constraint force profiles obtained with $a b$ initio constrained MD and PIMD simulations. The standard errors of these mean forces are smaller than $90 \mathrm{meV} / \AA$.
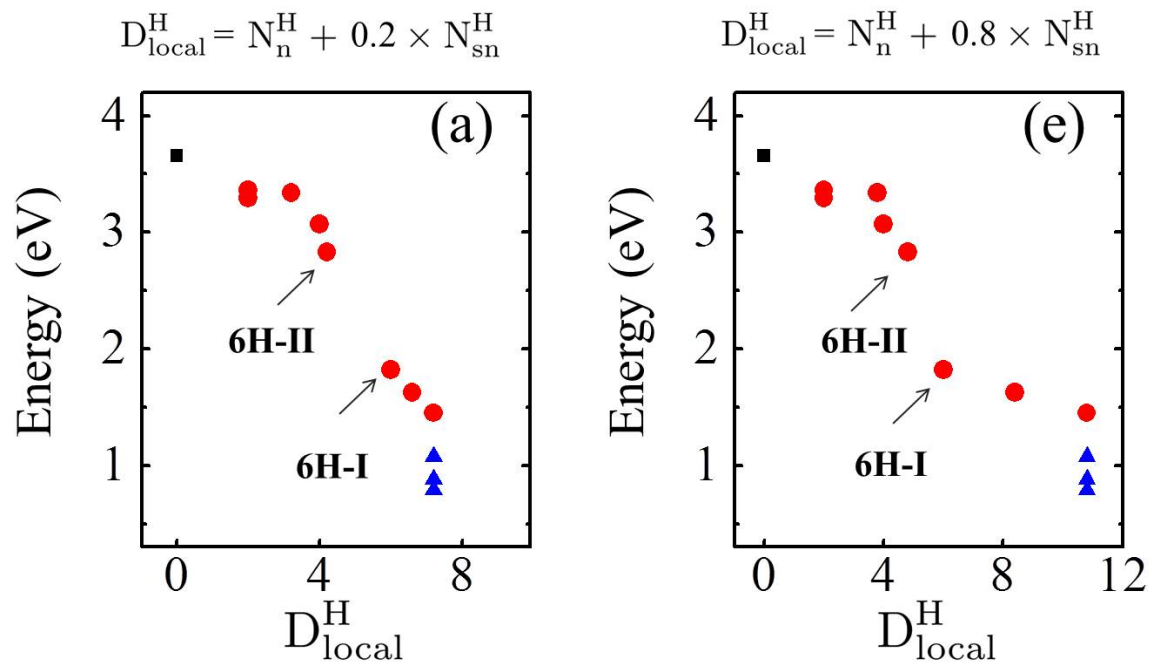

FIG. S11: The cNEB barriers for partially hydrogenated graphene, as a function of the local hydrogenation degree $\left(\mathrm{D}_{\text {local }}^{\mathrm{H}}\right)$, with different weight factor of 0.2 and 0.8 . 


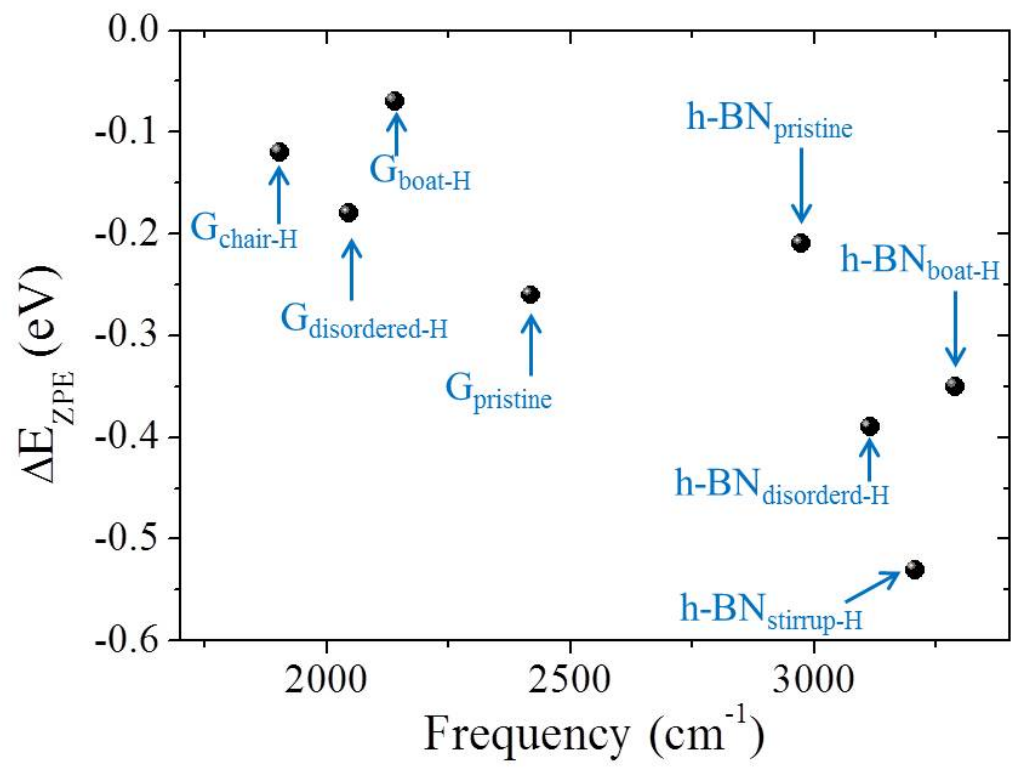

FIG. S12: The calculated ZPE correction $\left(\triangle E_{\mathrm{ZPE}}\right)$ along with the stretching frequencies of the covalent bonds between proton and $\mathrm{O}, \mathrm{C}$ and $\mathrm{N}$ atoms in the initial state of each system studied (pristine and hydrogenated 2D sheets). 

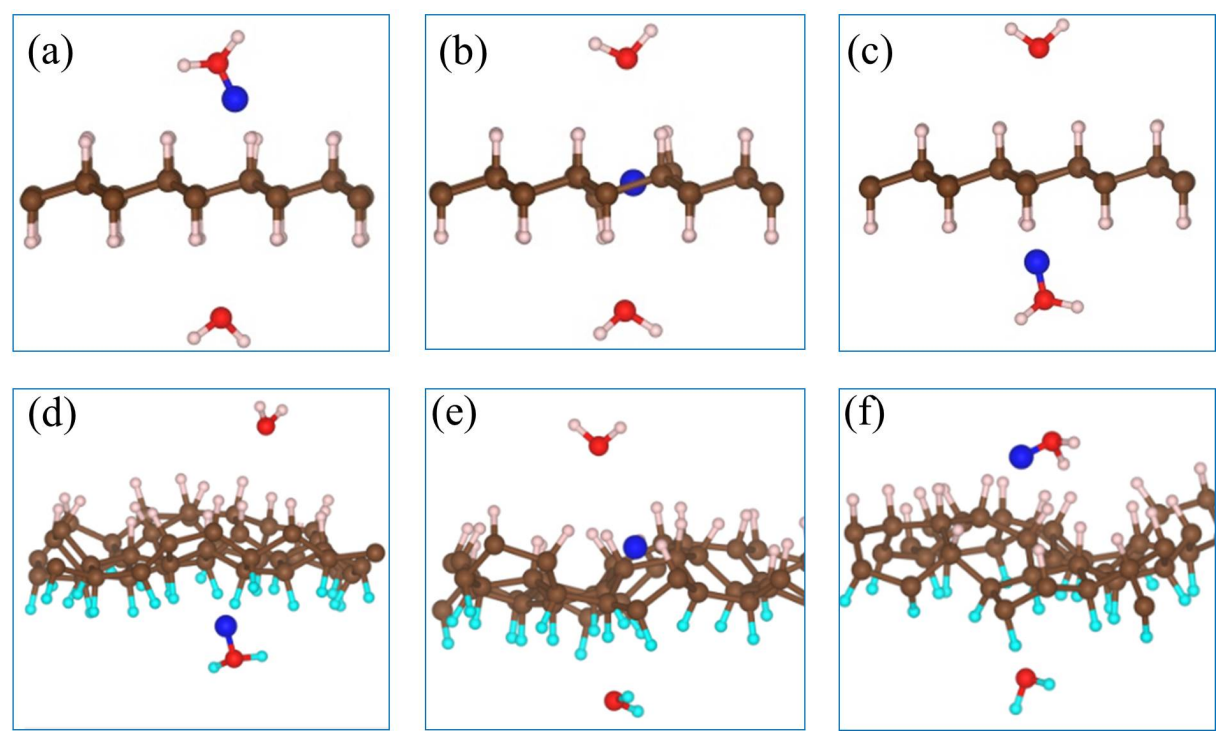

FIG. S13: The close-up views of the insets in the Fig. 4 of the main manuscript, which are the atomic structures for the initial, transition and final states for fully hydrogenated graphene (a)-(c) with the chair conformation and (d)-(f) with the disordered conformation. Red (pink, brown) balls are $\mathrm{O}(\mathrm{H}, \mathrm{C})$ atoms. Protons are represented by blue balls. The $\mathrm{H}$ adatoms below the sheets are colored with cyan (a contrast to pink) for clarity. 

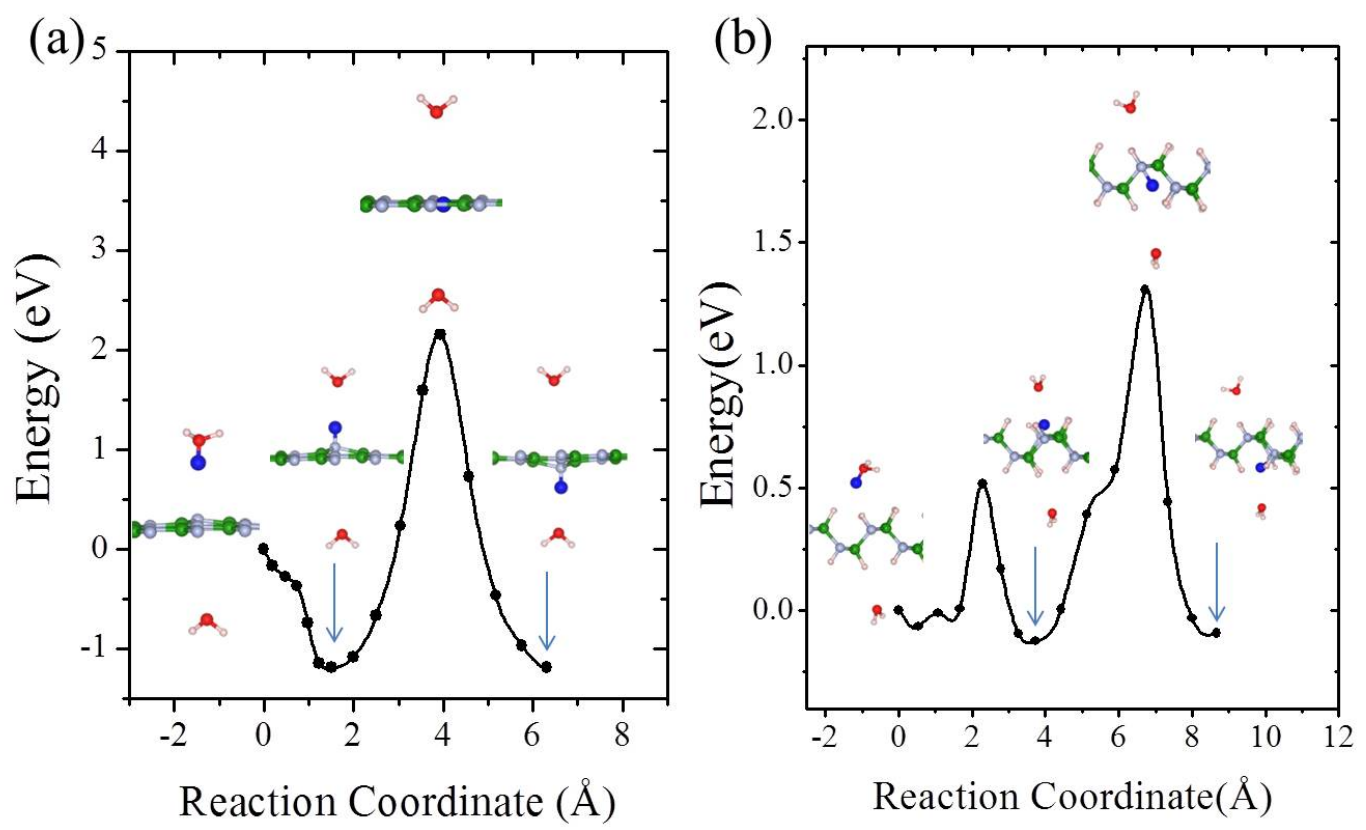

FIG. S14: Energy profiles for proton penetration across (a) pristine h-BN and (b) fully hydrogenated h-BN with the stirrup conformation, in the presence of water molecules. Insets show the atomic structures for some of the key states involved in the proton penetration process. Red (pink, green, gray) balls are $\mathrm{O}(\mathrm{H}, \mathrm{B}, \mathrm{N})$ atoms. Protons are represented by blue balls. Hydrogenation results in the fact that the chemisorbed state is no longer deep-lying, as compared with the pristine h-BN. This effect holds for all hydrogenated structures discussed in this manuscript. 

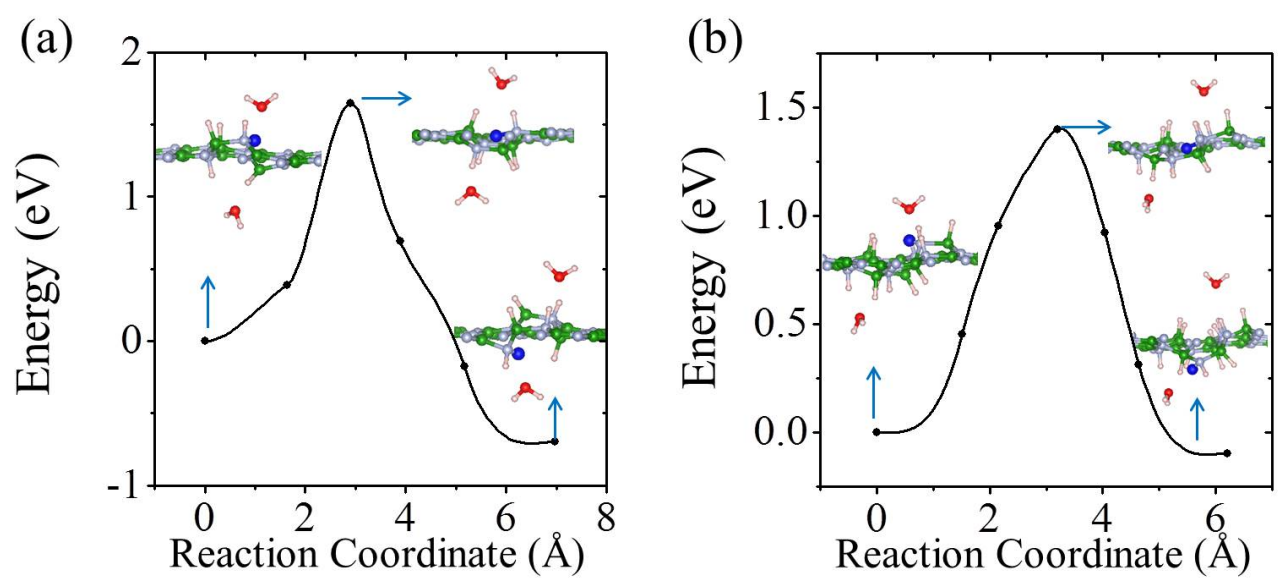

FIG. S15: Energy profiles for proton penetration across partially hydrogenated h-BN with $\mathrm{D}_{\text {local }}^{\mathrm{H}}$ equal to (a) 6 and (b) 9, respectively, when the weighting factor $\mathrm{w}$ is set to 0.5. Insets show the atomic structures for the initial, transition and final states. Red (pink, brown, green, gray) balls are $\mathrm{O}(\mathrm{H}, \mathrm{C}, \mathrm{B}, \mathrm{N})$ atoms. Protons are represented by blue balls. 


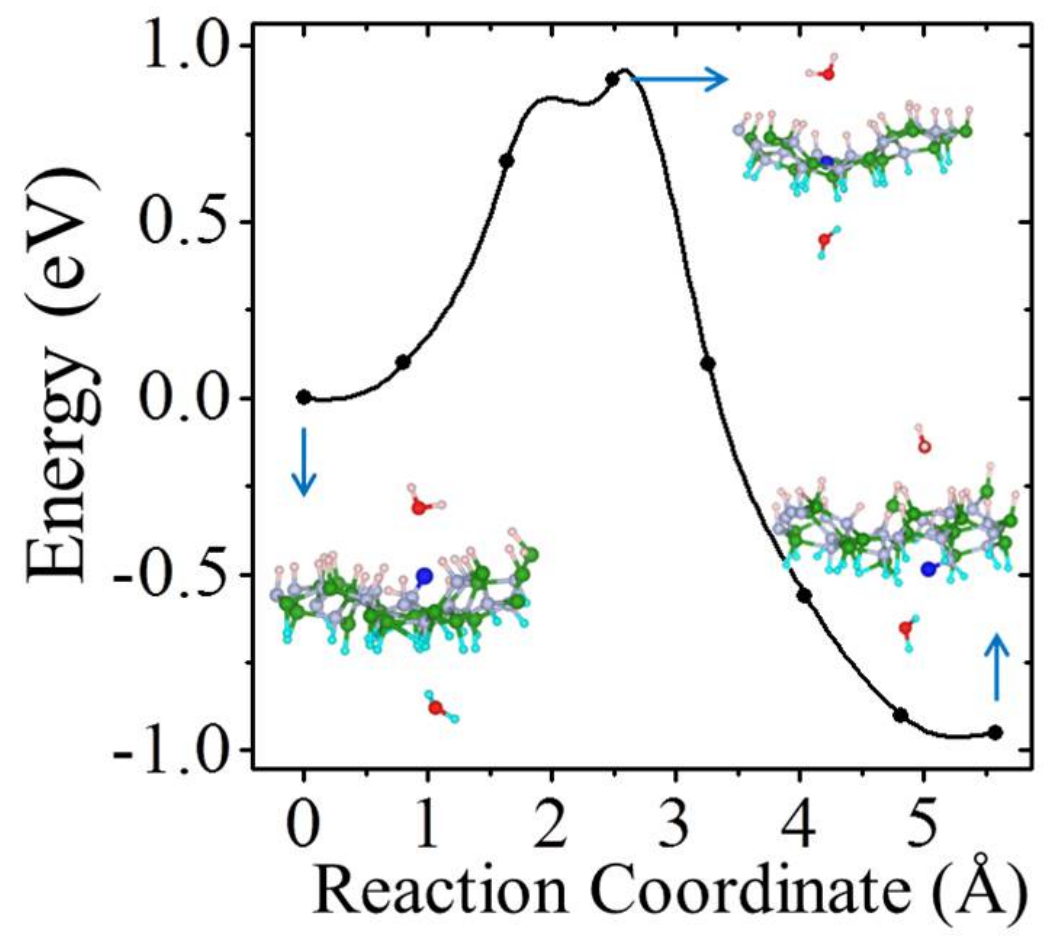

FIG. S16: Energy profiles for proton penetration across fully hydrogenated h-BN with a disordered $\mathrm{H}$ configuration. Insets show the atomic structures for the initial, transition and final states. Red (pink, brown, green, gray) balls are O (H, C, B, N) atoms. Protons are represented by blue balls. The $\mathrm{H}$ adatoms below the sheets are colored with cyan (a contrast to pink) for clarity. 